\title{
Statins in Chronic Kidney Disease- Are Statins Really Renoprotective
}

\author{
Sanjay Mehra ${ }^{1}$ and Tejas Desai ${ }^{2 *}$
}

${ }^{1}$ Department of Cardiovascular Sciences, East Carolina University- Brody School of Medicine, USA

${ }^{2}$ Division of Nephrology and Hypertension, East Carolina University- Brody School of Medicine, USA

\begin{abstract}
Numerous trials have shown that chronic kidney disease increases the risk of cardiovascular disease. Dyslipidemias have been shown to accelerate progression of kidney disease. Statins have assumed a pivotal role in management of hyperlipidemia. However their effect on renal function is still not fully understood. Some clinical trials have shown some renoprotective effect of statins but there is still need for more data to clarify effects of statins on renal function.
\end{abstract}

Keywords: Chronic kidney disease; Cardiovascular disease; Atherosclerosis; Dyslipidemia; Proteinuria

\section{Burden of Chronic Kidney Disease (CKD)}

According to the National Kidney Foundation figures, more than 8 million people in the United States have CKD. The leading cause of death in patients with CKD is cardiovascular disease (CVD) and accounts for $40 \%$ deaths in patients with end-stage kidney disease. $[1,2]$ Patients with CKD have an increased risk for CVD than those with normal renal function due to co-existing proteinuria, anemia, increased oxidative stress, inflammation and electrolyte imbalances. [3-5] Chade and colleagues studied coronary flow reserve in patients with CKD without obstructive coronary artery disease and found a reduced vasodilatory capacity in the coronary arteries compared with healthy controls. [6] Researchers have also found that carotid artery intima-media thickness is significantly greater in patients with CKD [7].

Evidence suggests that dyslipidemia contributes to progression of kidney disease and kidney disease progresses more rapidly in patients with hypercholesterolemia and hypertriglyceridemia compared to patients with normal lipid profile due to direct toxic effects of lipids and inflammatory pathways related to dyslipidemia and atherosclerosis. [810] The Helsinki Heart study aimed to document an association between progressive kidney disease and dyslipidemia. The study enrolled 2702 middle-aged dyslipidemic men and showed deterioration of renal function by $3 \%$ over 5 years and the decline was $20 \%$ faster in men with ratio of LDL/HDL > 4.4 compared with men with a ratio of LDL/ HDL < 3.2. [11] Dyslipidemia may contribute to early and accelerated vascular changes during early stages of renal impairment. In addition to accelerated atherosclerosis, lipid accumulation in renal cells upregulates intracellular signaling pathways which lead to a cascade of inflammatory and fibrogenic pathways and result in progressive renal impairment $[7,12]$.

\section{Mechanism of Action of Statins}

Statins have been proven to decrease the risk of cardiovascular disease and mortality. Despite that, only one third of patients with CKD and ESRD are currently being treated for hyperlipidemia. Possible causes could be lack of evidence of safety of statins in advanced kidney disease or lack of evidence supporting benefit of statins in patients with advanced CKD. [13] Various studies have been done to this effect to evaluate effect of statins on renal function and study their benefits in subjects with renal dysfunction. Statins act by inhibiting 3-hydroxy3-methylglutaryl coenzyme A (HMG-CoA) reductase, an enzyme vital for cholesterol synthesis and this inhibition leads to decreased mevalonate production and serum levels of low density lipoprotein cholesterol (LDL-C) decline. Statins exert anti-inflammatory and immunomodulatory effects by inhibiting mevalonate synthesis, which is a precursor for isoprenoids that are required for cellular pathways essential for inflammation and immune functions. Statins have been shown to decrease levels of tumor necrosis factor- $\alpha$, interleukin- 6 , transforming growth factor- $\beta$, vascular cell adhesion molecule-1, fibronectin mRNA and mesangial proteins. [14-16] In addition statins increase bioavailability of Nitric oxide, which is a potent vasodilator with apparent anti-inflammatory effects. [17] Statins also decrease oxidative stress by impeding oxidation of LDL. This anti-oxidant effect of statins enhances endothelial function $[18,19]$.

Fried and colleagues performed a meta-analysis of 13 small, prospective clinical trials evaluating effects of anti-hyperlipidemic drugs, mainly statins, on proteinuria, albuminuria and renal function. Lipid therapy was shown to significantly retard the rate of decline in GFR compared to controls [20].

A subgroup analysis of the Greek atorvastatin and coronary heart disease evaluation (GREACE) study reported the long-term effect of structured versus usual care and effect of statin versus no hypolipidemic drug treatment on estimated creatinine clearance $(\mathrm{CrCl})$. The eligible subjects in this study with established coronary heart disease (CHD) were randomized either into the structured group where they were started on atorvastatin at $10 \mathrm{mg} /$ day starting dose and followed by standard care University clinic or into the usual care group where they were followed by their general practitioners or cardiologists. In the structured care group the dose of atorvastatin was titrated up to $80 \mathrm{mg} /$ day to achieve the national cholesterol education program (NCEP) LDL-C goal ( $<2.6 \mathrm{~mol} / \mathrm{liter} ; 1000 \mathrm{mg} / \mathrm{liter})$. In the usual care group subjects were treated according to their physician's standard care which included life style changes, lipid lowering drugs and other necessary drug treatment. Subjects were followed for a mean three year period, with visits every six months. At baseline the subjects in either group had no significant differences in CHD risk factors.

On analysis of the usual care group, 687 subjects not on long term hypolipidemic drug therapy showed a $5.3 \%$ mean decline in

*Corresponding author: Tejas Desai, Division of Nephrology and Hypertension, East Carolina University- Brody School of Medicine, 2355 West Arlington Blvd, Greenville, USA, Tel: 252-744-1380; E-mail: desait@ecu.edu

Received August 13, 2011; Accepted October 05, 2011; Published October 13 2011

Citation: Mehra S, Desai T (2011) Statins in Chronic Kidney Disease- Are Statins Really Renoprotective. J Nephrol Therapeutic 1:103. doi:10.4172/21610959.1000103

Copyright: (c) Mehra S, et al. This is an open-access article distributed under the terms of the Creative Commons Attribution License, which permits unrestricted use, distribution, and reproduction in any medium, provided the original author and source are credited. 
$\mathrm{CrCl}(\mathrm{p}<0.0001)$. This was comparable to a mean decline of $4.9 \%$ in $\mathrm{CrCl}$ in 17 patients in the structured group who discontinued atorvastatin $(\mathrm{p}=0.02)$. Forty one subjects in the usual care group who were on simvastatin showed a mean $5.2 \%$ increase in $\mathrm{CrCl}(\mathrm{p}=0.002)$ and 25 subjects on atorvastatin showed a mean increase of $8.1 \%$ in $\mathrm{CrCl}(\mathrm{p}=0.0003)$. Pravastatin and fluvastatin treated subjects showed no statistically significant increase in their $\mathrm{CrCl}$. In the structured group, 783 subjects on atorvastatin showed a mean increase of $12 \%$ in $\mathrm{CrCl}$ $(p<0.0001)$. This beneficial effect was greatest in those subjects with early renal dysfunction. Figure 1 shows a breakdown of $\mathrm{CrCl}$ in either group at baseline and at 48 months. Figure 2 shows the mean percent change in $\mathrm{CrCl}$ in either group with or without statin therapy.

In the same study $292 \mathrm{CHD}$ related events were recorded, out of which 196 occurred in subjects on usual care and 96 on patients on atorvastatin. A multivariate analysis of $\mathrm{CHD}$ related events in the subjects revealed a hazard ratio (HR) of 1.10 [95\% confidence interval (CI), 1.03to 1.21; $\mathrm{p}=0.01$ ] with each $5 \%$ decline in $\mathrm{CrCl}$ and a $\mathrm{HR}$ of 1.18 (95\% CI, 1.09 to 1.29 ; $\mathrm{p}<0.001)$ with each $10 \%$ decline in $\mathrm{CrCl}$. In contrast a $5 \%$ increase in $\mathrm{CrCl}$ showed a $\mathrm{HR}$ of $0.84(95 \% \mathrm{CI}, 0.73$ to $0.95 ; \mathrm{p}=0.003)$ and a $10 \%$ increase in $\mathrm{CrCl}$ yielded a $\mathrm{HR}$ of $0.73(95 \% \mathrm{CI}$, 0.64 to $0.82 ; \mathrm{p}<0.001$ ) for $\mathrm{CHD}$ related events.

This study suggests a decline in renal function over a period of three years of follow-up in dyslipidemic patients with CHD who had normal renal function at baseline and were not treated with statins. The study also suggests that statins exert an inhibitory effect on decline in renal function and actually shows an improvement in renal function, as measured by $\mathrm{CrCl}$, in patients treated with statins in the same period. This improvement in renal function also correlates with a lower CHD related event rate with statin therapy [21].

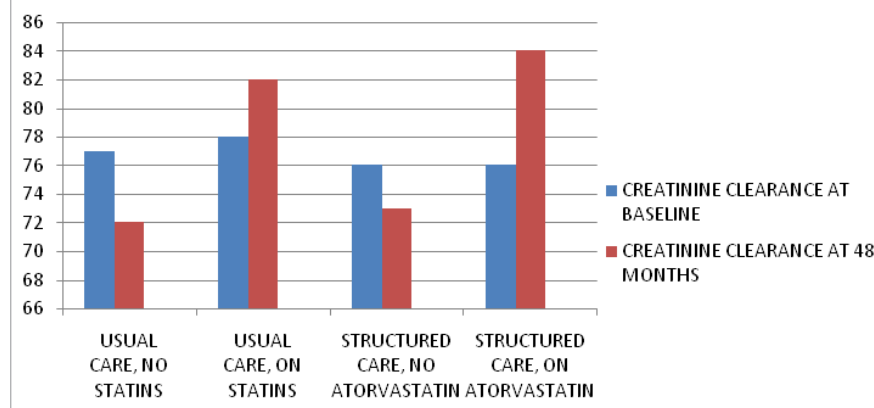

Figure 1: Comparison of creatinine clearance (mean values in $\mathrm{ml} / \mathrm{min}$ ) in the structured and usual care groups at baseline and at 48 months. Adapted from Athyros et al. 2004.

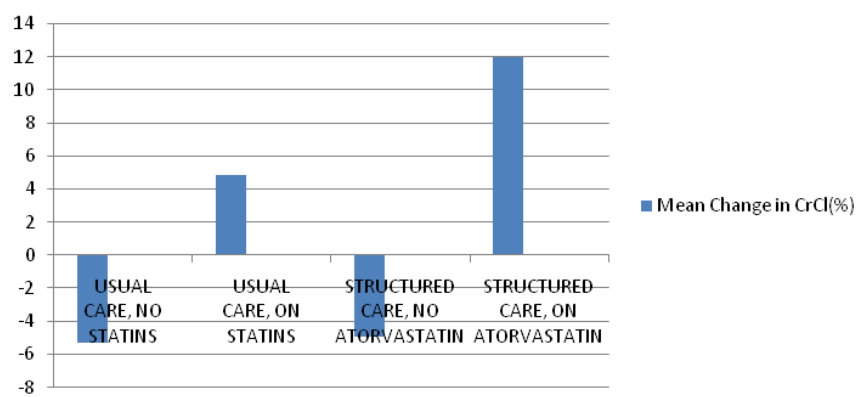

Figure 2: Mean change in $\mathrm{CrCl}$ in the structured and usual care groups after 48 months. Adapted from Athyros et al. 2004.

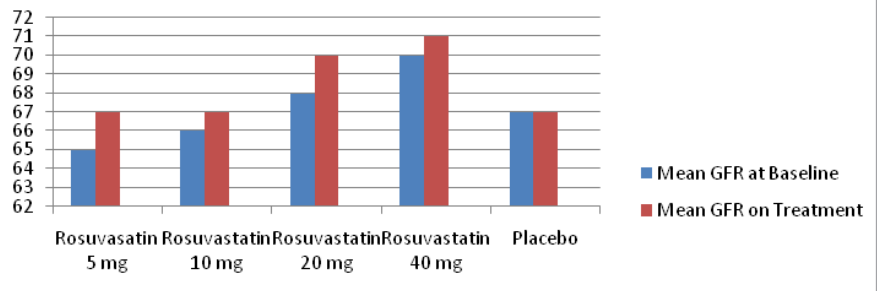

Figure 3: Comparison of change in GFR $\left(\mathrm{ml} / \mathrm{min} / 1.73 \mathrm{~m}^{2}\right)$ in short -term controlled clinical trials in patients on placebo versus patients on different doses of rosuvastatin. Adapted from Vidt et al. 2004.

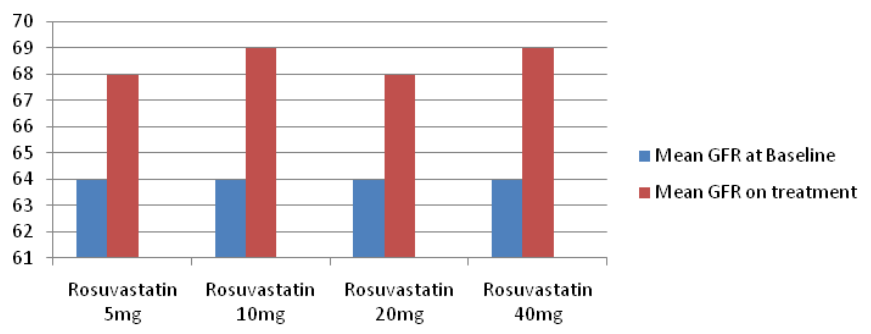

Figure 4: Comparison of change in GFR $\left(\mathrm{ml} / \mathrm{min} / 1.73 \mathrm{~m}^{2}\right)$ in patients on longterm treatment with rosuvastatin. Adapted from Vidt et al. 2004.

Vidt and colleagues performed a pooled analysis of data from a population of hyperlipidemic patients enrolled in the rosuvastatin clinical development program to assess the effect of rosuvastatin on renal function. The study group was initially evaluated in shortterm controlled clinical trials which compared efficacy and safety of rosuvastatin with other lipid lowering drugs or placebo. Subsequently patients were permitted to participate in an open-label extension trial, where patients received rosuvastatin in the recommended dose range (5-40 $\mathrm{mg}$ ) for up to 3.8 years. Renal function was measured by serum creatinine and GFR, which was obtained from the Modification of Diet in Renal Disease (MDRD) equation. The Glomerular filtration rate (GFR) was noted to be higher than baseline both early and later in the course of rosuvastatin treatment, with no change seen in placebo group as depicted in Figure 3. This study revealed that the mean GFR after 96 weeks of treatment with rosuvastatin was unchanged or higher when compared with the GFR at baseline irrespective of age, sex, presence of hypertension or diabetes and level of renal function at baseline. Results are depicted in Figure 4.

The notable finding in this study was absence of a decline in renal function in patients receiving long-term rosuvastatin therapy. Infact a renoprotective effect of rosuvastatin was observed most notably in patient with pre-existing kidney disease. Another observation of this study was a dip-stick positive proteinuria in a small percentage of rosuvastatin-treated patients. This is attributed to a decrease in tubular absorption of albumin as seen with any statin [22].

Strippoli and colleagues performed a meta-analysis of 50 randomized and quasi-randomized controlled trials of statins compared with other statins or placebo in CKD. Statins were found to significantly reduce total cholesterol, LDL-C and proteinuria. However the analysis did not support an improvement of GFR with statins. Another significant finding was reduced fatal and non-fatal cardiovascular events with statin therapy without any significant effect on all cause mortality. Relatively sparse data and possible reporting bias on outcomes left questions regarding renoprotective effects of statins uncertain [23]. 


\section{Statins Use in Kidney Transplant Patients}

Assessment of Lescol in Renal Transplant trial enrolled 2102 renal transplant patients with total cholesterol of $155-348 \mathrm{mg} / \mathrm{dl}$ and they were randomly assigned to fluvastatin $40 \mathrm{mg}$ or $80 \mathrm{mg}$ versus placebo and then followed for 5-6 years. Patients in the fluvastatin treatment arm showed a $32 \%$ decrease in LDL-C and reduction in the risk for major adverse cardiac events. However there was no improvement seen in the incidence of renal graft loss or doubling of serum creatinine or less deterioration in the GFR over time compared to placebo [24].

Two recent trials that have attempted to provide definitive evidence of a possible renoprotective action and safety of statins in kidney disease are PLANET I (Prospective evaluation of proteinuria and renal function in diabetic patients with progressive renal disease) and PLANET II (Prospective evaluation of proteinuria and renal function in non-diabetic patients with progressive renal disease). PLANET I and PLANET II both aimed to compare effects of rosuvastatin (10 and $40 \mathrm{mg}$ ) and atorvastatin $(80 \mathrm{mg})$ on reducing urinary protein excretion, renal function and lipid parameters. The results of these trials were recently reported at the XLVII European Renal AssociationEuropean Dialysis and Transplant Association Congress. In PLANET I atorvastatin was found to reduce proteinuria by $15 \%$ on top of angiotensin converting enzyme inhibitor/ angiotensin II receptor blocker therapy, with no significant effect seen by either dose of rosuvastatin. The results were the same for albuminuria with the two drugs. In PLANET II atorvastatin reduced proteinuria by more than $20 \%$ at 26 and 52 weeks, with no significant effect of either dose of rosuvastatin again. Interestingly there was a significant decline of estimated GFR (about $8 \mathrm{ml} / \mathrm{min} / 1.73 \mathrm{~m}^{2}$ ) noted in PLANET II with rosuvastatin at $40 \mathrm{mg} /$ day dose. No significant difference in the amount of lipid lowering was reported among the treatment groups [25-27].

\section{Recent Research}

The Study of heart and renal protection (SHARP) randomized more than 9000 patients to the combination of simvastatin and ezetimibe versus placebo and statin versus placebo. Study results published recently showed that patients allocated to take ezetimibe plus simvastatin had one-sixth fewer major atherosclerotic events, with similar reductions in all types of patients studied. Study showed a large reduction in LDL-C by addition of ezetimibe $10 \mathrm{mg}$ / day to simvastatin $20 \mathrm{mg} /$ day, with no evidence of increased risk for muscle or liver problems. Another trial that merits attention is a study to evaluate the use of rosuvastatin in subjects on regular hemodialysis: an assessment of survival and cardiovascular events (AURORA). This trial randomized 2776 hemodialysis patients to rosuvastatin versus placebo. Rosuvastatin therapy did result in a $43 \%$ reduction in LDL-C levels after 3 months of follow-up, but failed to show any significant effect on allcause mortality and on the primary end-point of the study, which was death from cardiovascular causes, non-fatal myocardial infarction or non-fatal stroke. Hopefully the results of these two trials may address some of the questions about effect of dyslipidemias on renal function and role of statins in patients with CKD [28-31].

The Treat to New Targets study was undertaken to investigate the effect of intensive lipid lowering with atorvastatin $80 \mathrm{mg}$ compared to atorvastatin $10 \mathrm{mg}$ on the risk of future cardiovascular events. The study population included 10,001 eligible men and women, aged 35-75 years with clinically evident CHD. All participants were taken off any lipid-lowering therapy they were on and subject to 1-8 weeks of washout period. Subsequently subjects with LDL-C between 130-250 mg/ $\mathrm{dl}$ and triglycerides less than $600 \mathrm{mg} / \mathrm{dl}$ were entered into an 8 week run-in period with treatment with atorvastatin $10 \mathrm{mg} /$ day. This phase was followed by randomization of subjects with LDL-C less than 130 $\mathrm{mg} / \mathrm{dl}$ to double-blind therapy with either atorvastatin $80 \mathrm{mg} /$ day or 10 $\mathrm{mg} /$ day for upto 6 years.

Occurrence of a major cardiovascular event was the primary efficacy outcome for this study and defined as death from CHD, nonfatal nonprocedure-related myocardial infarction, resuscitation after cardiac arrest, or fatal or non-fatal stroke. Results showed that patients with CKD were at a significantly greater risk for a major cardiovascular event than patients with normal renal function (Hazard Ratio 1.35; 95\% CI 1.18 to $1.54 ; \mathrm{p}<0.0001)$. Among patients with CKD at baseline, a first major cardiovascular event occurred in $9.3 \%$ receiving atorvastatin $80 \mathrm{mg} /$ day compared to $13.4 \%$ in patients receiving atorvastatin 10 $\mathrm{mg} /$ day, which translates into a $32 \%$ relative risk reduction( $\mathrm{p}=0.0003$ ). Another important inference from this study was that the rates of treatment-related adverse events and discontinuation rate due to treatment-related adverse effects were similar between patients with CKD and patients with normal estimated glomerular filtration rate, in either treatment group [32,33].

\section{Conclusion}

The National Kidney Foundation is urging physicians to consider $\mathrm{CKD}$ as a CVD risk equivalent due to an observed progressive increase in CVD risk with worsening kidney disease. [34] Statins have a proven role in reduction of LDL-C and cardiovascular morbidity and mortality. Their role in decreasing proteinuria and their renoprotective effect is currently under debate. CKD should not preclude use of a statin as recommended by National Lipid Association Statin safety Assessment Task Force. [35] PLANET I and PLANET II support effect of atorvastatin in reducing proteinuria but did not show any beneficial effect on renal function. The trials have raised questions regarding earlier views of class effect with statins as results with rosuvastatin differed from atorvastatin. We still have to await results from the ongoing research to uncover the effect of statins on renal function.

\section{References}

1. National Kidney Foundation (2006) K/DOQI clinical practice guidelines for chronic kidney disease: evaluation, classification, and stratification. Am J Kidney Dis 2002: 39-66.

2. Mason NA, Bailie GR, Satayathum S, Bragg-Gresham JL, Akiba T, et al (2005) HMG-Coenzyme A reductase inhibitor use is associated with mortality reduction in hemodialysis patients. Am J Kidney Dis 45:119-126.

3. Kidney Disease Outcomes Quality Initiative (K/DOQI) group (2003) K/DOQ clinical practice guidelines for managing dyslipidemias in chronic kidney disease. Am J Kidney Dis 41: 1-91.

4. Astor BC, Coresh J, Heiss G, Pettitt D, Sarnak MJ (2006) Kidney function and anemia as risk factors for coronary heart disease and mortality: The Atherosclerosis Risk in Communities (ARIC) study. Am Heart J 151: 492-500.

5. Go AS, Chertow GM, Fan D, Charles EM, Chi-yuan H (2004) Chronic kidney disease and the risks of death, cardiovascular events, and hospitalization. New Engl J Med 351: 1296-1305.

6. Chade AR, Brosh D, Higano ST, Lennon RJ, Lerman LO, et al. (2006) Mild renal insufficiency is associated with reduced coronary flow in patients with non-obstructive coronary artery disease. Kidney Intl 69: 266-271.

7. Preston E, Ellis MR, Kulinskaya E, Alun HD, Edwina AB (2005) Association between carotid artery intima-media thickness and cardiovascular risk factors in CKD. Am J Kidney Dis 46: 856-862.

8. Diamond JR, Karnovsky MJ (1988) Focal and segmental glomerulosclerosis: analogies to atherosclerosis. Kidney Int 33: 917-924.

9. Kasiske BL, O'Donnell MP, Cowardin W, Keane WF (1990) Lipids and the kidney. Hypertension 15: 443-450. 
10. Schaeffner ES, Kurth T, Curhan GC, Glynn RJ, Rexrode KM, et al. (2003) Cholesterol and the risk of renal dysfunction in apparently healthy men. J Am Soc Nephrol 14: 2084-2091.

11. Manttari M, Tiula E, Alikoski T, Vesa Manninen (1995) Effects of hypertension and dyslipidemia on the decline in renal function. Hypertension 26: 670-675.

12. Oda H, Keane WG (1999) Recent advances in statins and the kidney. Kidney Int Suppl 71: 2-5.

13. Fox CS, Longenecker JC, Powe RN, Klag MJ, Fink NE, et al. (2004) Under treatment of hyperlipidemia in a cohort of United states kidney dialysis patients. Clin Nephrol 61: 299-307

14. Mason JC (2003) Statins and their role in vascular protection. Clin Sci (London) 105: $251-266$

15. Crisby M (2003) Modulation of the inflammatory process by statins. Drugs Today ( Barcelona) 39: 137-143.

16. Pierre-Paul D, Gahtan V (2003) Noncholesterol-lowering effects of statins. Vasc Endovascular Surg 37: 301-313

17. Wolfrum S, Jensen KS, Liao JK (2003) Endothelium-dependant effects of statins. Arterioscler Thromb Vasc Biol 23:729-736.

18. Laufs U, La Fata V, Plutzky J, James KL (1998) Upregulation of endothelial nitric oxide synthase by HMG CoA reductase inhibitors. Circulation 97: 11291135

19. Hernández-Perera O, Pérez-sala D, Navarro-Antolín, Sánchez-Pascuala R, Hernández GJ, et al. (1998) Effects of the 3-hydroxy-3-methylglutarylCoA reductase inhibitors, atorvastatin and simvastatin, on the expression of endothelin-1 and endothelial nitric oxide synthase in vascular endothelial cells. J Clin Invest 101: 2711-2719.

20. Fried LF, Orchard TJ, Kasiske BL (2001) Effect of Lipid reduction on the progression of renal disease: a meta-analysis. Kidney Int 59: 260-269.

21. Athyros VG, Mikhailidis DP, Papageorgiou AA, Symeonidis AN, Pehlivanidis AN, et al. (2004) The Effect of statins versus untreated dyslipidemia on rena function in patients with coronary heart disease. A subgroup analysis of the Greek atorvastatin and coronary heart disease evaluation (GREACE) study. $J$ Clin Pathol 57: 728-734.
22. Vidt DG, Cressman MD, Harris S, Hutchinson HG (2004) Rosuvastatin-Induced Arrest in Progression of Renal Disease. Cardiology 102:52-60.

23. Strippoli GFM, Navaneethan SD, Johnson DW, Perkovic V, Pellegrini F, et al (2008) Effects of statins in patients with chronic kidney disease: meta-analysis and meta-regression of randomized controlled trials. BMJ 336: 645-651.

24. Fellstrom B, Holdaas H, Jardine AG, Holme I, Nyberg G, et al. (2004) Effect of fluvastatin on renal end points in the assessment of lescol in renal transplant (ALERT) trial. Kidney Int 66: 1549-1555.

25. PLANET I Prospective evaLuation of proteinuriA and reNal function in diabETic patients with progressive renal disease (D3569C00007).

26. PLANET II (2010) Prospective evaLuation of proteinuriA and reNal function in non-diabETic patients with progressive renal disease (D3569C00011).

27. Keller DM (2010) Atorvastatin Beats Rosuvastatin in Protecting Kidneys in Diabetic and Nondiabetic Patients.

28. United States Renal Data System (2007) Mortality and cause of death.

29. Baigent C, Landry M (2003) Study of heart and renal protection (SHARP) Kidney Int 84: 07-10.

30. SHARP: Ezetimibe plus statin reduced CV risk in patients with CKD. Endocrine Today.

31. Fellström BC, Jardine AG, Holdaas H, Schmieder RE, Bannister K, et al. (2009) Rosuvastatin and Cardiovascular Events in Patients Undergoing Hemodialysis. N Engl J Med 1395-1407.

32. Shepherd J, Kastelein JJP, Bittner V, Deedwania P, Breazna A, et al. (2008) Intensive Lipid Lowering with Atorvastatin in Patients with Coronary Hear Disease and Chronic Kidney Disease: The TNT (Treating to New Targets) Study. J Am Coll Cardiol 51: 1448-1454.

33. LaRossa JC, Grundy SM, Waters DD, Charles Shear, Philip Barter, et al. (2005) Intensive Lipid Lowering with atorvastatin in patients with stable coronary disease. N Engl J Med 352:1425-1435.

34. Anavekar NS, McMurray JJV, Velazquez EJ, Solomon SD, Kober L, et al. (2004) Relation between renal dysfunction and cardiovascular outcomes after myocardial infarction. New Engl J Med 351: 1285-1295.

35. Kasiske BL, Wanner C, O'Neill WC (2006) An assessment of statin safety by nephrologists. Am J Cardiol 97: 82-85. 